\title{
Modeling of radio-frequency induced currents on lead wires during MR imaging using a modified transmission line method
}

\author{
Volkan Acikel ${ }^{\mathrm{a})}$ and Ergin Atalar \\ Department of Electrical and Electronics Engineering, Bilkent University, Bilkent, Ankara 06800, Turkey \\ and National Magnetic Resonance Research Center (UMRAM), Bilkent, Ankara 06800, Turkey
}

(Received 30 June 2011; revised 20 October 2011; accepted for publication 25 October 2011; published 23 November 2011)

Purpose: Metallic implants may cause serious tissue heating during magnetic resonance (MR) imaging. This heating occurs due to the induced currents caused by the radio-frequency (RF) field. Much work has been done to date to understand the relationship between the RF field and the induced currents. Most of these studies, however, were based purely on experimental or numerical methods. This study has three main purposes: (1) to define the RF heating properties of an implant lead using two parameters; (2) to develop an analytical formulation that directly explains the relationship between RF fields and induced currents; and (3) to form a basis for analysis of complex cases.

Methods: In this study, a lumped element model of the transmission line was modified to model leads of implants inside the body. Using this model, leads are defined using two parameters: impedance per unit length, $Z$, and effective wavenumber along the lead, $k_{t}$. These two parameters were obtained by using methods that are similar to the transmission line theory. As long as these parameters are known for a lead, currents induced in the lead can be obtained no matter how complex the lead geometry is. The currents induced in bare wire, lossy wire, and insulated wire were calculated using this new method which is called the modified transmission line method or MoTLiM. First, the calculated induced currents under uniform electric field distribution were solved and compared with method-of-moments (MoM) calculations. In addition, MoTLiM results were compared with those of phantom experiments. For experimental verification, the flip angle distortion due to the induced currents was used. The flip angle distribution around a wire was both measured by using flip angle imaging methods and calculated using current distribution obtained from the MoTLiM. Finally, these results were compared and an error analysis was carried out.

Results: Bare perfect electric, bare lossy, and insulated perfect electric conductor wires under uniform and linearly varying electric field exposure were solved, both for $1.5 \mathrm{~T}$ and $3 \mathrm{~T}$ scanners, using both the MoTLiM and MoM. The results are in agreement within 10\% mean-square error. The flip angle distribution that was obtained from experiments was compared along the azimuthal paths with different distances from the wire. The highest mean-square error was $20 \%$ among compared cases.

Conclusions: A novel method was developed to define the RF heating properties of implant leads with two parameters and analyze the induced currents on implant leads that are exposed to electromagnetic fields in a lossy medium during a magnetic resonance imaging (MRI) scan. Some simple cases are examined to explain the MoTLiM and a basis is formed for the analysis of complex cases. The method presented shows the direct relationship between the incident RF field and the induced currents. In addition, the MoTLiM reveals the RF heating properties of the implant leads in terms of the physical features of the lead and electrical properties of the medium. (ㅇ 2011 American Association of Physicists in Medicine. [DOI: 10.1118/1.3662865]

Key words: MRI, implant safety, RF, induced currents, RF heating

\section{INTRODUCTION}

Magnetic resonance imaging (MRI) is an important diagnostic imaging tool. The main advantage of MRI is its ability to obtain high soft tissue contrast and resolution. MRI is a very safe imaging technique, except for patients with metallic implants. However, there is a high risk of serious radiofrequency (RF) heating and tissue damage due to the induced currents on leads of the implants. RF heating is the result of altered electric field distribution where a conductive wire exists. ${ }^{1}$ Much work has been done to understand the effect of induced currents on metallic wires inside the human body, ${ }^{2,3}$ most of which are based on experimental studies ${ }^{4}$ or numerical simulations. ${ }^{1,5,6}$ All of these works ${ }^{1,4,5}$ show standing wave behavior of current but none of them can formulate it. A solution that shows the relationship between the induced current on the wires and the position of the wire in the body, the wire dimensions and insulation thickness would help to understand the problem and also this may also be used in the design of novel leads. 
King has summarized an analytical formulation that was developed for the use of insulated antennas in sea water. ${ }^{7}$ In this approach, a dipole antenna in sea water was modeled as a transmission line with an infinite outer conductor. King showed that currents on dipole or monopole antennas exhibit traveling wave behavior. ${ }^{8}$ He stated that the current on a finite dipole or monopole can be represented as a superposition of traveling waves in both directions along the antenna. ${ }^{8}$ This traveling wave behavior is similar to the transmission line currents. ${ }^{9}$ In all of these works, antennas were examined inside highly conducting mediums. This kind of approach can be used to analyze induced currents on wires under an electromagnetic field inside a lossy medium. A similar modification was done by Przybyszewski to predict implant lead heating. ${ }^{10}$

Using the assumption that scattered fields from wire will decay quickly, King's ${ }^{7,8}$ approach can be used to analyze induced currents on metallic implants inside low-conductive tissues. In the modified transmission line method (MoTLiM), the lumped element model of transmission lines was modified by adding a voltage source to model the effect of an incident electric field. Using this modified lumped element model, a nonuniform differential equation is obtained for the current on the lead. The differential equation formulates the standing wave behavior of induced currents. In the nonuniform differential equation, two parameters, the impedance per unit length, $Z$, and effective wavenumber along the lead, $k_{t}$, govern the current.

In this study, the analytical solution under a uniform electric field distribution was determined. In addition, the required parameters for a perfect electric conductor wire, a lossy conductor wire and an insulated perfect electric conductor wire were derived. After the theory is stated, verification is provided by the results of simulations and experiments.

\section{THEORY}

In this study, a phasor notation with a time dependence of $e^{i \omega t}$, where $\omega$ represents the angular frequency and $i=\sqrt{-1}$, is used. The complex wavenumber is defined as $k=\omega \sqrt{\mu \epsilon-i \sigma / \omega}$, and $\epsilon, \mu$, and $\sigma$ are the permittivity, permeability, and conductivity of the medium, respectively. The intrinsic impedance of the medium is defined as $\eta=\sqrt{\mu /(\epsilon-i \sigma / \omega)}$.

\section{II.A. Modified transmission line theory}

It is well known that transmission lines can be modeled using a combination of series and parallel lumped elements, as shown in Fig. 1. ${ }^{9}$ Here, $Z$ and $Y$ are the series impedance and the parallel admittance per unit length, respectively.

It is well known that the current in transmission lines can be formulated using the Telegrapher's equation

$$
\frac{d^{2} I}{d z^{2}}+k_{t}^{2} I(z)=0
$$

where $k_{t}$ is $\sqrt{-Y Z}$. In this model, the current in a wire is defined as a function of position $z$. The solution to this equa-

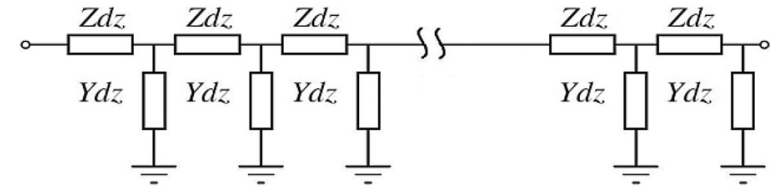

FIG. 1. The transmission line model using lumped circuit elements. $Z$ is the resistance and inductance per unit length of the transmission line. $Y$ is the admittance and capacitance per unit length of the transmission line. $d z$ is the length of an infinitesimal piece of the transmission line.

tion results in the well known solution of the transmission line theory

$$
I(z)=I_{+} e^{-i k_{t} z}+I_{-} e^{i k_{t} z} .
$$

The transmission line theory does not use a current or voltage source in its model. These sources may be used at the terminals of the transmission line in the form of boundary conditions.

For a wire in a lossy medium that is exposed to an electromagnetic wave, excitation is distributed along the length of the wire, and therefore, without modification, the transmission line model cannot be applied.

We propose to solve this problem by introducing a series voltage source and defining the shunt admittance in between the wire and the environment as shown in Fig. 2. Note that this model is not exact and as it will be formulated later, an approximate formulation will be obtained using this model. The assumption of fast decaying scattered fields must hold to be accurate. The model in Fig. 2 can be formulated using the following equation:

$$
I(z)+\frac{1}{k_{t}^{2}} \frac{d^{2} I(z)}{d z^{2}}=\frac{E^{i}(z)}{Z},
$$

where $E^{i}$ is the tangential component of the incident electric field, $k_{t}=\sqrt{-Z Y}$ is the effective wavenumber along the wire, and $Z$ is the distributed impedance. In this model, the wire diameter is assumed to be significantly smaller than the wavelength, and therefore, the electric field on the wire can be defined. The voltage source in Fig. 2 models the effect of the incident field. Another difference from the traditional transmission line method is the way in which $Z$ and $k_{t}$ parameters are found. This will be explained by the examples below.

\section{II.B. Solution of the current under uniform electric field}

Birdcage coils have a fairly uniform $E$-field distribution along their main axis; ${ }^{11}$ thus, implants exposed to uniform $E$-field distribution represent an important and likely

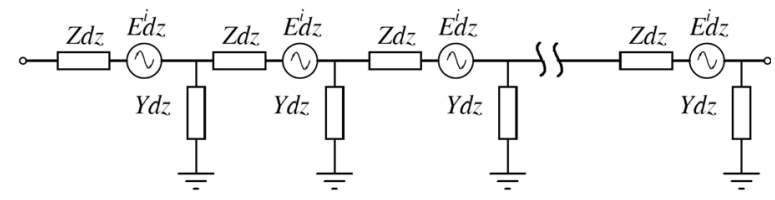

FIG. 2. The modified transmission line model using lumped circuit elements that includes a series voltage source. Here, the ground is assumed to be at the outer boundary of body with an infinite extent and $d z$ is the length of an infinitesimal piece of the wire. 
situation. In this section, currents induced on wires under a uniform $E$-field are determined.

Assume a wire with length $l$ and radius $a$ is located on the $z$-axis. To find the current on the wire, Eq. (3) must be solved. For the specific case in which the incident $E$-field is uniform along the wire, $E=E_{z 0} \hat{z}$ the solution to the Eq. (3) current can be found to be the following:

$$
I(z)=A e^{-i k_{t} z}+B e^{i k_{t} z}+\frac{E_{z 0}}{Z},
$$

where $k_{t}$ and $Z$ will be defined in Sec. II D. The unknowns $A$ and $B$ can be found by assuming that the lead current vanishes at both ends of the wire

$$
\begin{aligned}
& I(z=l / 2)=0 \\
& I(z=-l / 2)=0 .
\end{aligned}
$$

As a result, the current on the wire is found to be

$$
I(z)=\frac{E_{z 0}}{Z}-2 \frac{E_{z 0}}{Z} \frac{\sin \left(k_{t} \frac{l}{2}\right)}{\sin \left(k_{t} l\right)} \cos \left(k_{t} z\right) .
$$

\section{II.C. Solution of the current under linearly varying electric field}

In the central region of a birdcage coil, $E$-field distribution increases linearly in radial direction and has constant phase. Thus, it is a likely situation that implants exposed to linearly varying $E$-field distribution. In this section, induced currents on the wire with length $l$, under linearly varying $E$-field are solved. Assume the tangential $E$-field along the wire is

$$
E=E_{t 0} l^{\prime}
$$

where $l^{\prime}$ is the position on the wire and ranging from $-l / 2$ to $l / 2$. For this $E$-field exposure, solving Eq. (3) while applying the boundary conditions [Eq. (5)], induced currents on the wire can be found as

$$
I\left(l^{\prime}\right)=\frac{E_{t 0} l^{\prime}}{Z}-\frac{E_{t 0} l}{2 Z \sin (k t l / 2)} \sin \left(k t l^{\prime}\right) .
$$

As can be seen from this example case, Eq. (3) is the basis for solving any complex form of problem as long as the tangential component of the $E$-field along the length of the wire is known.

\section{II.D. Determination of $Z$ and $k_{t}$ parameters}

As seen in Eq. (6), the induced current on a wire can be found if $Z$ impedance per unit length, $k_{t}$ effective wavenumber along the wire, and the incident $E$-field are known. Therefore, a wire can be characterized by $Z$ and $k_{t}$, which summarize the electrical parameters of the body and physical features of the wire. In the transmission line theory, the series impedance, $Z$, and the wavenumber, $k_{t}$, are calculated using relations for the stored energy per unit length and dissipated power per unit length. ${ }^{9}$ However, defining these stored energy and dissipated power relations along a wire are not trivial, so traditional ways of determining $Z$ and $k_{t}$ in the transmission line theory is not valid for the case presented and a new approach must be used to determine these parameters. In this section, a detailed electromagnetic analysis to determine $Z$ and $k_{t}$ will be carried out for some simple but important cases: (1) a perfect electric conductor bare wire; (2) a lossy bare wire; and (3) an insulated wire. However, for much more complex lead geometries, the required parameters may not be easy to calculate analytically. In such cases, some measurements may be necessary to determine the parameters.

\section{II.D.1. Perfect electric conductor bare wire}

Assume that an infinitely long perfect electric conductor bare wire with a radius of " $a$ " is placed on the $z$-axis and is exposed to a uniform radial incident wave with linear phase variation such that

$$
E_{z}^{i}=E_{z 0} J_{0}\left(k_{\rho} \rho\right) e^{-i \beta_{z} z} .
$$

where $E_{z 0}$ is the magnitude of the incident field and $J_{0}($.$) is$ the 0th order Bessel function. In this case, the induced current on the wire, $I$, will be uniform along the length of the wire, and the scattered fields can be written as follows: ${ }^{12}$

$$
\begin{aligned}
& E_{z}^{s}=\left(A H_{0}^{(1)}\left(k_{\rho} \rho\right)+B H_{0}^{(2)}\left(k_{\rho} \rho\right)\right) e^{-i \beta_{z} z} \\
& E_{\rho}^{s}=i \frac{\beta_{z}}{k_{\rho}}\left(A H_{1}^{(1)}\left(k_{\rho} \rho\right)+B H_{1}^{(2)}\left(k_{\rho} \rho\right)\right) e^{-i \beta_{z} z} \\
& H_{\phi}^{s}=i \frac{1}{\eta}\left(A H_{1}^{(1)}\left(k_{\rho} \rho\right)+B H_{1}^{(2)}\left(k_{\rho} \rho\right)\right) e^{-i \beta_{z} z} .
\end{aligned}
$$

$H_{z}^{s}=0, H_{\rho}^{s}=0, E_{\phi}^{s}=0$, where $\mathrm{A}$ and $\mathrm{B}$ are the constants to be determined by boundary conditions and $H_{n}^{(1)}(),. H_{n}^{(2)}($.$) are$ Hankel functions of the first and second kind, respectively.

Scattered fields must be vanished at infinity. Because $H_{n}^{(1)}($.$) goes to infinity as its argument goes to infinity, A$ must be zero. Also, at the surface of the conductor, the tangential electric field component must be zero; ${ }^{13}$ thus, $E_{z}$ must be equal to $-E_{z 0} J_{0}\left(k_{\rho} \rho\right) e^{-i \beta_{z} z}$. Therefore, the constant $B$ can be determined to be

$$
\begin{aligned}
& E_{z}^{s}=E_{z 0}\left(-\frac{J_{0}\left(k_{\rho} a\right)}{H_{0}^{(2)}\left(k_{\rho} a\right)} H_{0}^{(2)}\left(k_{\rho} \rho\right)\right) e^{-i \beta_{z} z}, \\
& H_{\phi}^{s}=i E_{z 0} \frac{1}{\eta}\left(-\frac{J_{0}\left(k_{\rho} a\right)}{H_{0}^{(2)}\left(k_{\rho} a\right)} H_{1}^{(2)}\left(k_{\rho} \rho\right)\right) e^{-i \beta_{z} z} .
\end{aligned}
$$

On the surface of the conductor, the total tangential magnetic field is

$$
H_{\phi}^{t}(\rho=a)=i E_{z 0} \frac{1}{\eta}\left(J_{1}\left(k_{\rho} a\right)-\frac{J_{0}\left(k_{\rho} a\right)}{H_{0}^{(2)}\left(k_{\rho} a\right)} H_{1}^{(2)}\left(k_{\rho} a\right)\right) e^{-i \beta_{z} z}=i E_{z 0} \frac{1}{\eta}\left(\frac{J_{1}\left(k_{\rho} a\right) Y_{0}\left(k_{\rho} a\right)-J_{0}\left(k_{\rho} a\right) Y_{1}\left(k_{\rho} a\right)}{H_{0}^{(2)}\left(k_{\rho} a\right)}\right) e^{-i \beta_{z} z} .
$$


Applying Wronskian of Bessel functions ${ }^{14}$

$$
J_{n}(x) Y_{n}^{\prime}(x)-J_{n}^{\prime}(x) Y_{n}(x)=\frac{2}{\pi x},
$$

where ' denotes the derivative with respect to the entire argument of the Bessel function, Eq. (13) becomes

$$
H_{\phi}^{t}(\rho=a)=-\frac{2 E_{z 0}}{\eta k_{\rho} a \pi} \frac{1}{H_{0}^{(2)}\left(k_{\rho} a\right)} e^{-i \beta_{z} z}
$$

Using the magnetic field on the surface of the conductor, it becomes trivial to determine the current on the wire

$$
\begin{aligned}
I & =2 \pi a H_{\phi}^{t} \\
& =-\frac{4 E_{z 0}}{\eta k_{\rho}} \frac{1}{H_{0}^{(2)}\left(k_{\rho} a\right)} .
\end{aligned}
$$

Returning back to the MoTLiM described by Eq. (3), the incident electric field with a linear phase variation along the length of an infinitely long wire generates a current of the same form, i.e., $I=I_{0} \exp \left(-i \beta_{z} z\right)$. Therefore, our differential equation is transformed into the following:

$$
I+\frac{\beta_{z}^{2}}{k_{t, \text { bare }}^{2}} I=\frac{E_{z 0}}{Z_{\text {bare }}} .
$$

To find $Z$ and $k_{t}$, Eq. (16) is plugged in Eq. (17) and it is assumed that the field variation along the wire is small, i.e., $\beta_{z}<|k|$, and thus, $H_{0}^{(2)}\left(k_{\rho} a\right) \approx H_{0}^{(2)}(k a)$. By manipulating Eq. (16) and Eq. (17), $Z$ and $k_{t}$ can be calculated as follows:

$$
\begin{aligned}
& Z_{\text {bare }}=\frac{\eta k}{4} H_{0}^{(2)}(k a), \\
& k_{t, \text { bare }}=k .
\end{aligned}
$$

\section{II.D.2. Lossy conductor bare wire}

In Sec. II D 1, the analysis of a perfect electrical conductor wire was performed. Now assume that the wire has a finite conductivity $\sigma_{c}$.

As is known from the transmission line theory, $Z$, the impedance per unit length, includes loss of the conductor. Therefore, $Z$ and $k_{t}=\sqrt{-Z Y}$ must be redefined. For a lossy conductor wire, the impedance per unit length in the lumped element model could be defined as

$$
Z_{\text {lossy }}=Z_{\text {bare }}+Z_{\text {wire }},
$$

where $Z_{\text {lossy }}$ is impedance per unit length for a lossy conductor wire, $Z_{\mathrm{bare}}$ is impedance per unit length for a perfect electric conductor and $Z_{\text {wire }}$ is impedance due to the loss of the conductor. Assuming the radius of the wire is much larger than the skin depth, $Z_{\text {wire }}$ can be expressed as

$$
Z_{\text {wire }}=\frac{\sqrt{f \mu}}{2 a \sqrt{\pi \sigma_{c}}},
$$

where $f$ is frequency, $\mu$ is permeability, $\sigma_{c}$ is conductivity of wire, and $a$ is the radius of the wire. $Z_{\text {lossy }}$ can be written as follows:

$$
Z_{\text {lossy }}=\frac{\eta k}{4} H_{0}^{(2)}(k a)+\frac{\sqrt{f \mu}}{2 a \sqrt{\pi \sigma_{c}}} .
$$

If the radius of the wire is smaller than the skin depth, $Z_{\text {wire }}$ can be still used, but the skin depth may not be used to calculate $Z_{\text {wire. }}$. After $Z_{\text {lossy }}$ is known, $k_{t, \text { lossy }}$ can be defined as

$$
k_{t, \text { lossy }}=k_{t, \text { bare }} \sqrt{1+\frac{Z_{\text {wire }}}{Z_{\text {bare }}}} .
$$

\section{II.D.3. Insulated perfect electric conductor wire}

To analyze insulated perfect electric conductor wires, $Z$ and $k_{t}$ must be recalculated as in Sec. II D 1. To do this, the fields and boundary conditions, which were stated in Sec. II D 1 must be redefined. Because a dielectric material on the conductor exists, there must be two boundaries: one between the tissue and the dielectric material, at $\rho=b$, and one between the dielectric and the conductor, at location $\rho=a$. Parameters with the superscript $d$ are insulator parameters. Scattered fields inside the dielectric are

$$
\begin{aligned}
& E_{z s}^{d}=A^{d} H_{0}^{(1)}\left(k_{\rho}^{d} \rho\right)+B^{d} H_{0}^{(2)}\left(k_{\rho}^{d} \rho\right), \\
& H_{\phi s}^{d}=\frac{i}{\eta^{d}}\left(A^{d} H_{1}^{(1)}\left(k_{\rho}^{d} \rho\right)+B^{d} H_{1}^{(2)}\left(k_{\rho}^{d} \rho\right)\right),
\end{aligned}
$$

and the total fields inside the tissue are

$$
\begin{aligned}
& E_{z s}=A H_{0}^{(1)}\left(k_{\rho} \rho\right)+B H_{0}^{(2)}\left(k_{\rho} \rho\right), \\
& H_{\phi s}=\frac{i}{\eta}\left(A H_{1}^{(1)}\left(k_{\rho} \rho\right)+B H_{1}^{(2)}\left(k_{\rho} \rho\right)\right) .
\end{aligned}
$$

$H_{0}^{(1)}\left(k_{\rho} \rho\right)$ goes to infinity as $\rho$ goes to infinity; thus, the constant " $A$ " must be zero since both scattered electric and magnetic fields vanish at infinity. The remaining unknowns can be found by applying the electromagnetic boundary conditions. The total electric fields must be zero at the surface of the conductor and also be continuous at the coating and surrounding medium boundary. Moreover, the tangential components of the total magnetic fields must be continuous at the coating and medium boundary. By applying these boundary conditions, the unknowns can be determined. Finally, by using the fact that the tangential component of the magnetic field at the surface of the conductor must be equal to the current density and by making some simplifications and approximations, the induced current can be determined to be as follows:

$$
I=E_{z 0} \frac{8}{\pi k_{\rho} k_{\rho}^{d} b} \frac{1}{\eta^{d} H_{1}^{(2)}(k b) \frac{2}{\pi} \ln \frac{a}{b}-\eta H_{0}^{(2)}(k b) \frac{2}{\pi k_{\rho}^{d} b}} .
$$

When an infinitesimally small portion of wire is considered, the insulator can be thought of as a capacitor in series with $Y$ in the lumped element model. Thus, when insulating a wire only $Y$ will change. $Z$ will remain as in Eq. (18) for a wire with radius $b$.

$$
Z_{\text {insulated }}=\frac{\eta k}{4} H_{0}^{(2)}(k b) \text {. }
$$

Then, using Eq. (26) in Eq. (17), the propagation constant along the wire can be written as follows: 


$$
k_{t, \text { insulated }}=k \sqrt{-\frac{2 \eta H_{0}^{(2)}(k b)}{\pi k_{\rho}^{d} b \frac{1}{\eta^{d} H_{1}^{(2)}(k b) \frac{2}{\pi} \ln \frac{a}{b}-\eta H_{0}^{(2)}(k b) \frac{2}{\pi k_{\rho}^{d} b}}}} .
$$

\section{METHOD}

\section{III.A. Simulations}

For the case under uniform electric field exposure, the induced currents were solved using the MoTLiM for the straight bare perfect electric conductor, coated perfect electric conductor, and lossy conductor wire cases. These solutions were compared with electromagnetic field simulations carried out using FEKO (EM Software \& Systems Germany, Böblingen, $\mathrm{GmbH}$ ) simulations. For the simulations, a homogeneous body with an infinite extent was used. The relative permittivity and conductivity of the body was 81 and $0.42 \mathrm{~S} / \mathrm{m}$, respectively. The wire with radius $0.57 \mathrm{~mm}$ and length $0.25 \mathrm{~m}$ was located on the $z$-axis inside the uniform body with infinite extent. Both ends of the wire were floating inside the body. For simplicity, a plane wave excitation, with the $E$-field component along the $z$-direction, was applied such that the wire was exposed to a uniform electric field. The impedance and wavenumber of the wires used in the simulations and MoTLiM analysis were calculated using formulas in Sec. II D can be seen in Tables I and II.

Also the MoTLiM can be used for solving complex problems. To demonstrate this, the induced currents were solved for under linearly varying $E$-field exposure using both ғЕкО and the MoTLiM for the wire with same radius and length as in previous simulations.

For linearly varying $E$-field case, a birdcage coil was assumed for simulations. Field of the birdcage coil was approximated by summing four plane waves ${ }^{15}$ traveling in $\hat{x}$, $-\hat{x}, \hat{y}$, and $-\hat{y}$ directions with equal amplitude and appropriate phases. In this way, a circular magnetic field as well as the linearly varying $E$-field distribution are obtained around the central region. The wire with length of $0.25 \mathrm{~m}$ placed at the origin of the homogeneous body with angle of $10^{\circ}$ with the $z$-axis. In this case coordinates on the wire are $z=l^{\prime} \cos \alpha$, $x=l^{\prime} \sin \alpha$ and $y=0$, where $l^{\prime}$ is the position on the wire and ranging between $-l / 2$ and $l / 2$.

For the central region inside a birdcage coil, $E$-field can be written as

$$
E=\hat{z} E_{0} x,
$$

TABLE I. Impedance and effective wavenumber of wires for $64 \mathrm{MHz}$.

\begin{tabular}{lcc}
\hline \hline $64 \mathrm{MHz}$ & $\begin{array}{c}\text { Effective wavenumber } \\
\left(\mathrm{m}^{-1}\right)\end{array}$ & $\begin{array}{c}\text { Impedance per unit } \\
\text { length }(\Omega / \mathrm{m})\end{array}$ \\
\hline Bare pec wire & $14.2-i 7.5$ & $87.3+i 386$ \\
Bare lossy conductor wire & $13.8-i 9.4$ & $187.3+i 386$ \\
Insulated pec wire & $15.4-i 9.5$ & $87.3+i 386$ \\
\hline
\end{tabular}

TABLE II. Impedance and effective wavenumber of wires for $123 \mathrm{MHz}$.

\begin{tabular}{lcc}
\hline \hline $123 \mathrm{MHz}$ & $\begin{array}{c}\text { Effective wavenumber } \\
\left(\mathrm{m}^{-1}\right)\end{array}$ & $\begin{array}{c}\text { Impedance per } \\
\text { unit length }(\Omega / \mathrm{m})\end{array}$ \\
\hline Bare pec wire & $24.7-i 8.3$ & $193+i 670$ \\
Bare lossy conductor wire & $24.6-i 10.1$ & $293+i 670$ \\
Insulated pec wire & $26.2-i 9.6$ & $193+i 687$ \\
\hline \hline
\end{tabular}

where $E_{0}=50 \mathrm{~V} / \mathrm{m}$ for $3 \mathrm{~T}$ and $E_{0}=40 \mathrm{~V} / \mathrm{m}$ for $1.5 \mathrm{~T}$. Then, tangential $E$-field on the wire will be

$$
E_{t 0}=\frac{E_{0} l^{\prime}}{2} \sin (2 \alpha) \text {. }
$$

Then, plugging Eq. (30) into Eq. (8) solution of the induced current under linearly varying $E$-field incidence can be found.

\section{III.B. Experimental verification of MoTLiM}

Although the MoTLiM promises a good explanation and understanding of induced currents on metallic implant leads its validity must be determined by experiments. This can be done by measuring induced currents during an MRI scan. However, measuring induced currents via current probes without distorting the incident field, and consequently, the induced currents is a challenging task. ${ }^{16}$ There are other methods to measure induced currents; however, they require the modification of leads. ${ }^{16}$ In this study, an undesirable MRI phenomenon was used to measure induced currents. A uniform RF field is desired during an MRI scan, but in the presence of a wire, RF fields due to the induced current produces nonuniform RF fields, and consequently, a nonuniform flip angle ${ }^{17}$ distribution in the vicinity of the wire. This nonuniform flip angle distribution was measured using the double angle method ${ }^{18}$ in the vicinity of the wire. Also, the flip angle distribution inside the uniform phantom was measured to determine the incident fields. From the measured flip angle distribution incident $B_{1}$ field can be calculated and for the experimental setup that was explained below, tangential component of the incident $E$-field can be calculated as

$$
E_{i}=\omega \rho \frac{B_{1}}{2} \cos (\omega t) \text {. }
$$

After the incident fields were determined they were used to calculate the induced current via the MoTLiM. Next, the flip angle distribution around the wire was calculated using this solution.

During experiments, a body birdcage coil was used and a cylindrical phantom was placed inside the MR scanner, as shown in Fig. 3. With this geometry, the incident $E$-field along the wire can be assumed to be uniform. A cylindrical phantom with a diameter of $28 \mathrm{~cm}$ and height of $6.5 \mathrm{~cm}$ was used. The conductivity and relative permittivity values of the phantom were $0.42 \mathrm{~S} / \mathrm{m}$ and 81 , respectively, and were measured using a custom-made transmission line probe ${ }^{19}$ with an Agilent E5061A ENA series network analyzer. Experiments were conducted using a copper bare wire with a radius of $0.57 \mathrm{~mm}$. The wire was circulated to form a full 


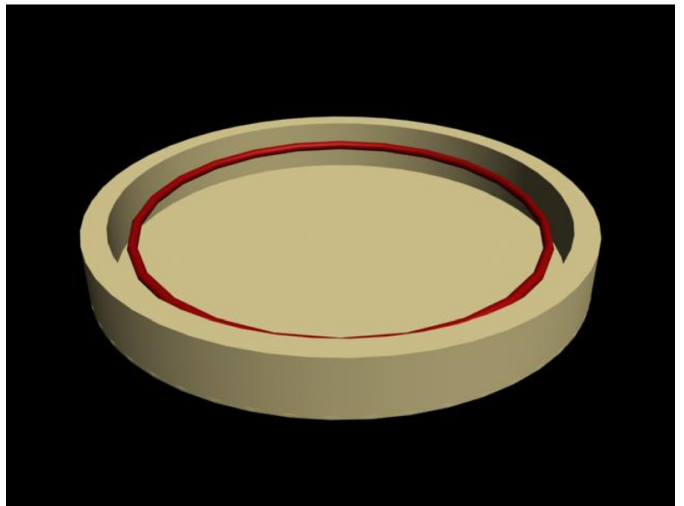

Fig. 3. Wire location inside phantom. The conductivity and relative permittivity of the phantom was $0.42 \mathrm{~S} / \mathrm{m}$ and 81 , respectively. The phantom was $28 \mathrm{~cm}$ in diameter and $6.5 \mathrm{~cm}$ high. The phantom was a solution of $\% 0.2$ copper sulfate $\% 0.1$ sodium chloride $\% 1.5$ hydroxyethyl cellulose. A circulated bare copper wire with radius $0.57 \mathrm{~mm}$ was used. The wire was located on a circle with a $12 \mathrm{~cm}$ radius and $3.2 \mathrm{~cm}$ above the bottom of the phantom. The phantom was located such that the center of the phantom coincided with the center of the body birdcage coil.

circle with a $12 \mathrm{~cm}$ radius and it was placed at the location $3.2 \mathrm{~cm}$ from the bottom of the phantom. Experiments were performed using a 3T Siemens TimTRIO system. A spin echo (SE) sequence with TR of $1000 \mathrm{~ms}$, TE of $15 \mathrm{~ms}$ and FOV of $300 \times 65 \mathrm{~mm}^{2}$ was used to acquire an axial image at the center of the phantom.

During experiments a body birdcage coil was used as a transmitting and receiving coil and was assumed to be ideal. For an ideal birdcage coil, the incident magnetic field can be written as follows:

$$
B_{1 f}^{c}=B_{1}(\cos (\omega t) \hat{x}-\sin (\omega t) \hat{y})
$$

For this specific experimental setup configuration, shown in Fig. 3, only the $y$ component of the incident magnetic field produces a flux on the surface encircled by the wire. Therefore, determining the $y$ component of the incident field is sufficient to determine the induced current on the wire.

Using some electromagnetic principles and vector algebra, the total forward polarized magnetic field can be found to be

$$
\left|B_{\text {total }}\right|=\sqrt{\left|B_{1}\right|^{2}+\frac{\left|B^{w}\right|^{2}}{4}+\left|B_{1}\right|\left|B^{w}\right| \sin \left(\theta^{w}-\phi\right)},
$$

where $\theta^{w}$ is the phase difference between $B_{1}$ and the current, $\phi$ is the azimuthal angle, $B_{1}$ is the incident field, and $B^{w}$ is the field caused by the induced currents. This total $B$ field expression can be used to calculate the flip angle.

\section{RESULTS}

To verify the proposed method, currents were solved using the MoTLiM and compared with electromagnetic field simulations. As mentioned before, the MoTLiM was also tested experimentally by measuring and calculating the flip angle distribution caused by induced currents.

\section{IV.A. Simulation results}

For the straight bare perfect electric conductor, coated perfect electric conductor, and lossy conductor wire cases the induced currents were solved both using the MoTLiM and FEKO and were compared as shown in Fig. 4. For the bare perfect electric conductor wire, mean-square errors were $8 \%$ and $6 \%$ for $3 \mathrm{~T}$ [Fig. 4(a)] and $1.5 \mathrm{~T}$ [Fig. 4(b)] scanners, respectively. For the coated perfect electric conductor wire, case mean-square errors were $4 \%$ and $7 \%$ for $3 \mathrm{~T}$ [Fig. 4(c)] and $1.5 \mathrm{~T}$ [Fig. 4(d)] scanners, respectively. And for the bare lossy conductor wire, case mean-square errors were $8 \%$ and $6 \%$ for $3 \mathrm{~T}$ [Fig. 4(e)] and $1.5 \mathrm{~T}$ [Fig. 4(f)] scanners, respectively. Also, induced currents on the wire were solved under linearly varying $E$-field incidence. Mean-square errors were $8 \%$ and $9 \%$ for $3 \mathrm{~T}$ [Fig. 5(a)] and $1.5 \mathrm{~T}$ [Fig. 5(b)] scanners, respectively.

\section{IV.B. Experimental results}

Next, experimental verification of the MoTLiM was done using flip angle images that were obtained both experimentally and theoretically. During the experiments, this configuration ensured that the wire was exposed to a uniform electric field. Flip angle distributions were measured and calculated using the data obtained from the MoTLiM formulation. Error analysis was performed along circles with different radii $(4-16 \mathrm{~cm})$ around the wire. The mean-square errors are between $16 \%$ and $20 \%$. Image artifacts not only depend on the magnitude of the induced current but are also affected by the phase of the induced current. As shown in Fig. 6, the artifact has constructive and destructive effects on the intensity of the image. The location of the destructive and constructive parts depends on the phase of the induced currents. Figure 7 also shows that phase of the current calculated using the MoTLiM accurately.

\section{DISCUSSION AND CONCLUSION}

In this study, a new method was developed to solve for the induced currents on leads inside a bodily tissue. To asses the validity of this method, some simple cases (straight perfect electric conductor bare, lossy conductor wire, and insulated perfect electric conductor wire) were solved and compared with the results of computer simulations that used method-of-moments. These simulations was done under uniform $E$-field incidence and as a more complex case bare perfect electric conductor wire was solved under linearly varying $E$-field incidence. During the simulations, only a straight bare perfect electric conductor, lossy conductor, and insulated perfect electric conductor wires were solved. These analyses revealed mean-squared error less than 10\%. As shown in Sec. II D to derive $Z$ and $k_{t}$ parameters field expressions were written for an infinitely long wire with uniform current. However, during the simulations the wire had finite length and was not long enough so that the induced current could not reach the steady state value. For the regions where the currents are changing infinitely, long wire assumptions cause errors. Also, for the insulated perfect electric wire 

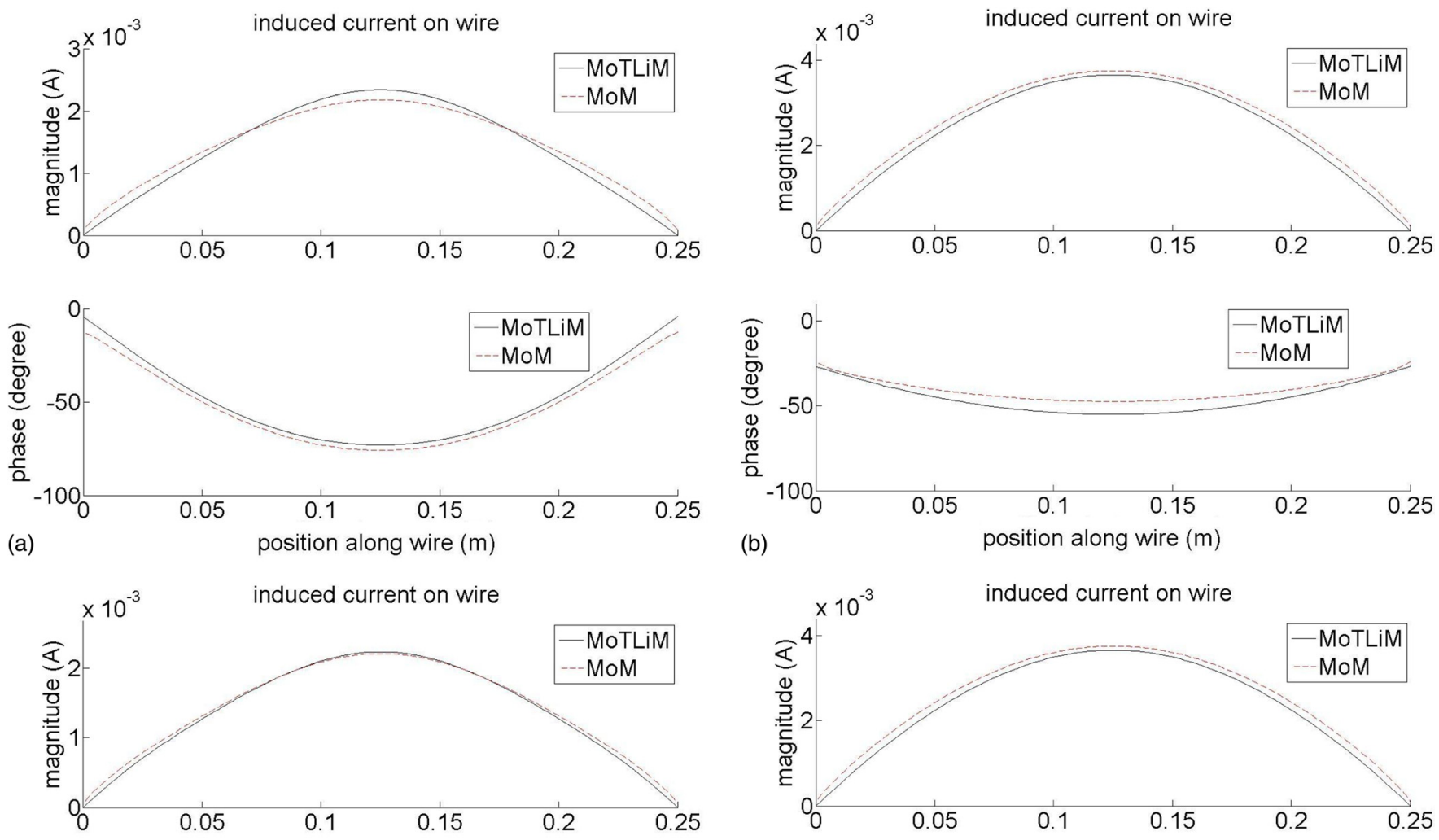

(b)

position along wire $(\mathrm{m})$
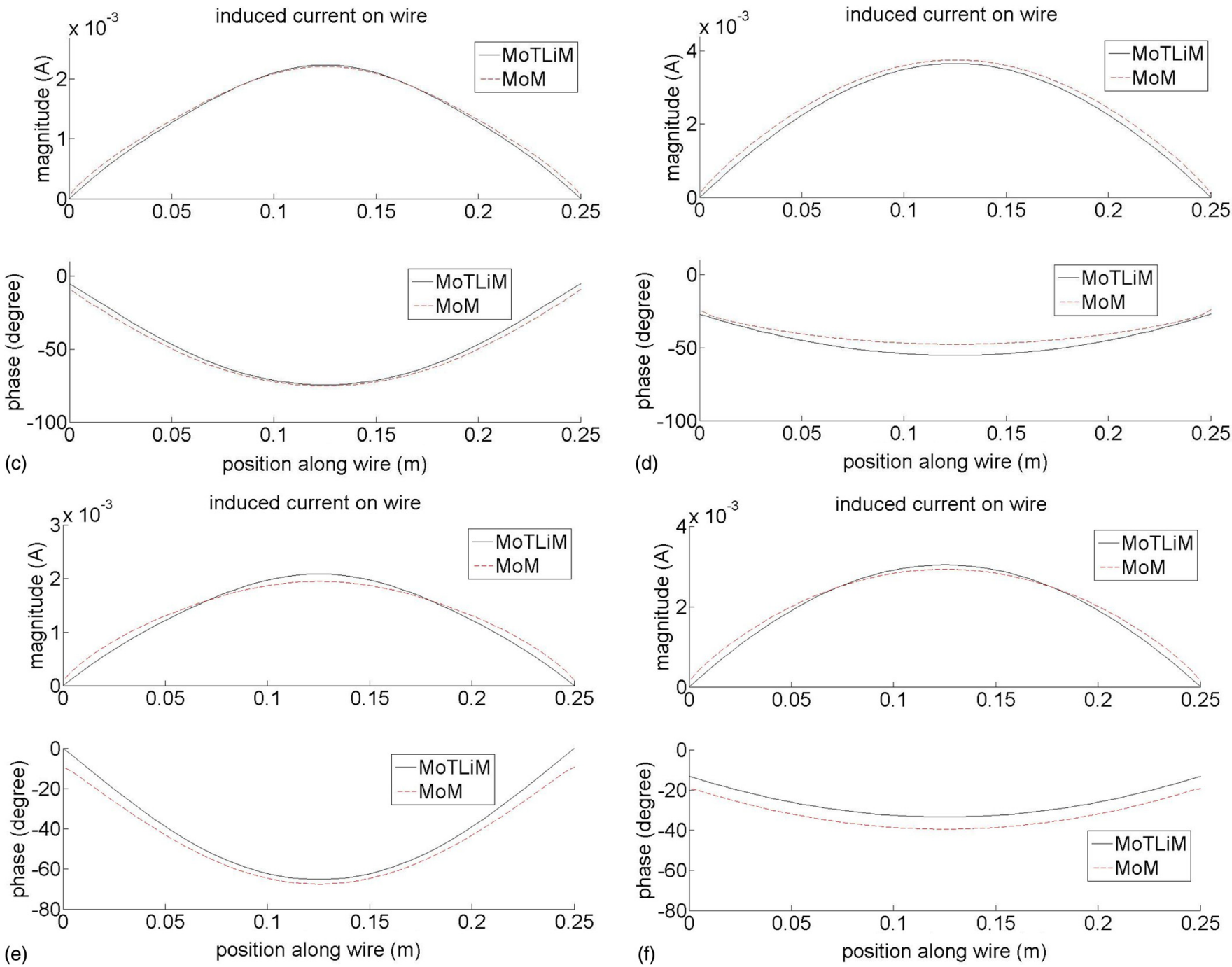

Fig. 4. Induced current on a wire with length $0.25 \mathrm{~m}$ and radius $0.57 \mathrm{~mm}$. (a) at $123 \mathrm{MHz}$ and (b) at $64 \mathrm{MHz}$ for a bare wire, (c) at $123 \mathrm{MHz}$ and (d) at $64 \mathrm{MHz}$ for a coated wire with a coating thickness of $5 \mu \mathrm{m},(\mathrm{e})$ at $123 \mathrm{MHz}$ and (f) at $64 \mathrm{MHz}$ for a lossy bare wire (100 $\Omega / \mathrm{m}$ resistance). A wire under uniform $E$-field $(1 \mathrm{~V} / \mathrm{m}$ ) exposure was solved using both FEKо (EM Software \& Systems Germany, Böblingen, GmbH) and the MoTLiM. In the simulations, the wire was located inside a lossy medium with an infinite extent. The medium possessed a conductivity and relative permittivity of $0.42 \mathrm{~S} / \mathrm{m}$ and 81 , respectively. Solid lines are the MoTLiM results and dashed lines are the FEKO results. 

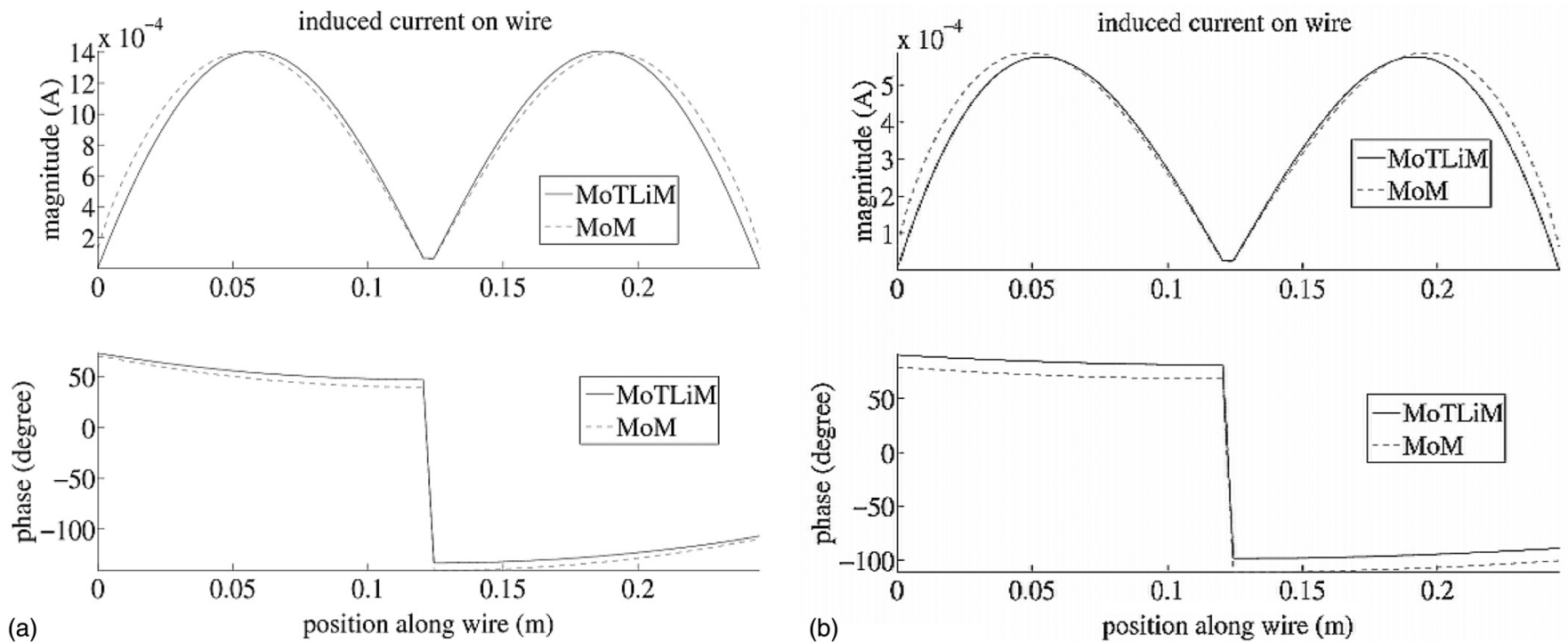

Fig. 5. Induced current on a wire with length $0.25 \mathrm{~m}$ and radius $0.57 \mathrm{~mm}$. (a) at $123 \mathrm{MHz}$ and (b) at $64 \mathrm{MHz}$ for a bare wire. A wire under linearly varying E-field exposure was solved using both FEKO and the MoTLiM. In the simulations, the wire was located inside a lossy medium with an infinite extent. The medium possessed a conductivity and relative permittivity of $0.42 \mathrm{~S} / \mathrm{m}$ and 81 , respectively. Solid lines are the MoTLiM results and dashed lines are the FEKO results.

case, the insulation thickness must be small such that the fields inside the coating material do not change.

We also compared the performance of this method with the results of a phantom experiment as described earlier. As shown in Fig. 7, the experimental and theoretical flip angle results are also in good agreement, and the mean-square error is less than $20 \%$.

In this work, the relationship between heating and the induced currents on the lead have not been studied. To predict the tip heating of a lead, relationship between induced currents and heating must be solved using bioheat transfer formulation. ${ }^{1}$ Also, for the analysis of heating of medical leads, electrodes must be modeled. Modeling an electrode as an impedance may be convenient for the MoTLiM, however, it needs further study.

With the MoTLiM formulation, it is rather straightforward to calculate the induced currents for different lead lengths and different electric field exposures. For example, recently, transmit array systems were introduced as an alternative means of transmitting RF pulses. These systems can change the electric field dynamically; therefore, this situation makes calculations rather complex. Our new MoTLiM formulation offers a straightforward solution even for these complex cases.

Although, in this study, only simple lead designs were solved this method may be used to solve more complicated

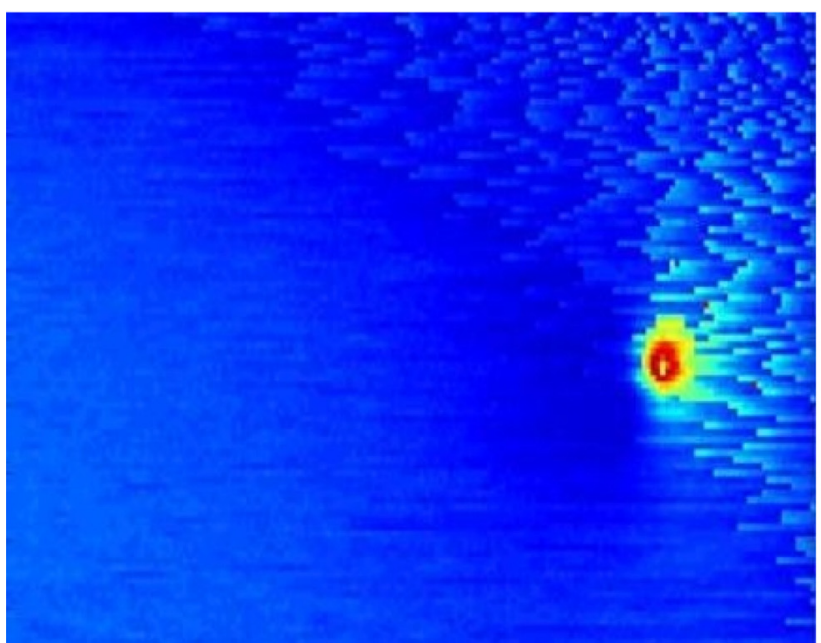

(a)

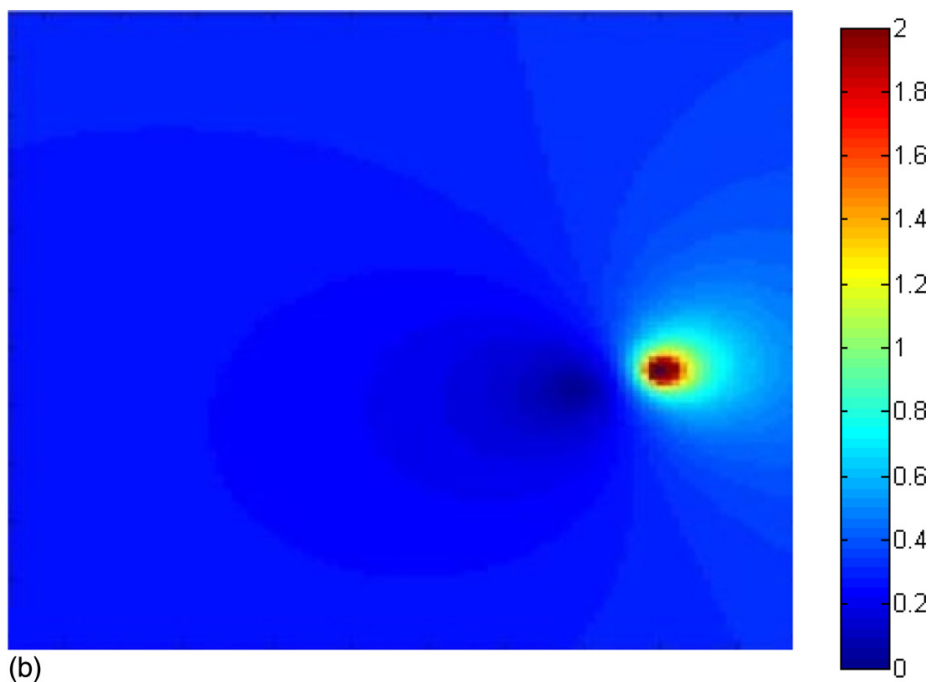

(b)

FIG. 6. Flip angle images when $1 \mathrm{~V}$ nominal is applied to transmit coil. (a) is the flip angle distribution obtained using DAM. (b) is the flip angle distribution calculated theoretically, as explained in Sec. III B. Flip angle distribution was calculated for a $60 \times 100 \mathrm{~mm}^{2}$ part of $300 \times 65 \mathrm{~mm}^{2}$ image and theoretical calculations are done for the same part. Calculated and measured flip angle distributions are presented using the same color scale in degrees. 

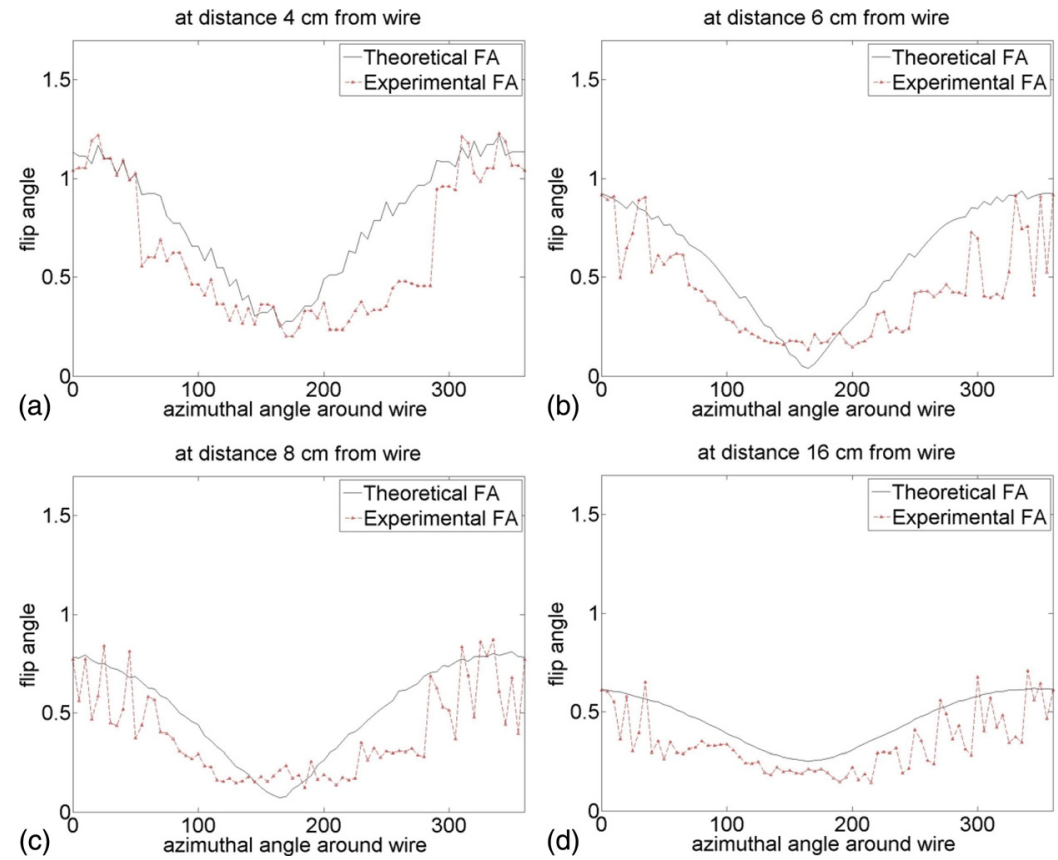

FIG. 7. Flip angle distribution on a circle, centered on a wire with radius (a) $4 \mathrm{~cm}$, (b) $6 \mathrm{~cm}$, (c) $8 \mathrm{~cm}$, and (d) $16 \mathrm{~cm}$. Flip angle values are in degree. Mean-square errors are $16 \%, 20 \%, 19 \%$, and $18 \%$ for (a), (b), (c), and (d), respectively.

designs. The solution of more complex cases like coiling or billabong winding requires further study. To solve these kinds of complex cases, the effective wavenumber and impedance per unit length must be defined. Moreover to complete presented study, electrodes must be modeled. If electrodes can be modeled as electrical loads, the current on the lead terminated with an electrode may be determined by redefining the boundary conditions. Also, using this theory, the effect of adding lumped elements along the lead (such as series inductors ${ }^{21}$ ) on the current distribution along the lead may be formulated. Although this study constitutes a basis to analyze these complex cases, they all remain as future work.

The MoTLiM may have applications beyond the heating of implants during MRI, such as in cell phone implant interactions. There are studies that have analyzed SAR gain in the presence of a deep brain stimulator ${ }^{22}$ during the use of cell phones. The MoTLiM may also be used to calculate induced currents on these implants when they interact with cell phones.

In conclusion, the presented formulation forms a basis for determining the impedance per unit length and effective wavenumber of implant leads. Using this formulation, the standing wave behavior of currents on the lead can be formulated in a similar manner as that of a transmission line. This formulation can be used to understand the worst-case heating amount and conditions.

\section{ACKNOWLEDGMENTS}

Special thanks to Emre Kopanoglu for his helpful comments. This work is partially supported by TUBITAK $107 \mathrm{E} 108$

\footnotetext{
a) Author to whom correspondence should be addressed. Electronic mail: vacik@ee.bilkent.edu.tr

${ }^{1}$ C. J. Yeung, R. C. Susil, and E. Atalar, "RF safety of wires in interventional MRI: Using a safety index,” Magn. Reson. Med. 47, 187193 (2002).
}

${ }^{2}$ M. K. Konings, L. W. Bartels, H. F. M. Smits, and C. J. G. Bakker, "Heating around intravascular guidewires by resonating RF waves," J. Magn. Reson. Imaging 12, 7985 (2000).

${ }^{3}$ F. G. Shellock, "Radiofrequency energy-induced heating during MR procedures: A review," J. Magn. Reson. Imaging 12, 3036 (2000).

${ }^{4}$ P. A. Bottomley, W. A. Edelstein, A. Kumar, M. J. Allen, and P. Karmarkar, "Resistance and inductance based MRI-safe implantable lead strategies," 17th Annual ISMRM Meeting, Honolulu, Hawaii, April 18-24 (2009).

${ }^{5}$ C. J. Yeung, P. Karmarkar, and R. M. Elliot, "Minimizing RF heating of conducting wires in MRI," Magn. Reson. Med. 58, 1028-1034 (2007).

${ }^{6}$ S.-M. Park, "MRI safety: Radiofrequency field induced heating of implanted medical devices," Ph.D. thesis, Purdue University, 2006.

${ }^{7}$ R. W. P. King, "The many faces of the insulated antenna," Proc. IEEE 64(2), 228-238 (1976)

${ }^{8}$ L. C. Shen, T. T. Wu, and R. W. P. King, "A simple formula of current in dipole antennas," IEEE Trans. Antennas Propag. 16(5), 542-547 (1968).

${ }^{9}$ D. M. Pozar, Microwave Engineering, 3rd ed. (John Wiley \& Sons, New York, 2005).

${ }^{10}$ P. Przybyszewski, M. Wiktor, and M. Mrozowski, "Modeling of pacing lead electrode heating in the MRI RF field," Proceedings of the 17th International Zurich Symposium on Electromagnetic Compatibility (EMC Zurich 2006), Singapore, 27 February-3 March 2006.

${ }^{11} \mathrm{~J}$. Jianming, Electromagnetic Analysis and Design in Magnetic Resonance Imaging (CRC, 1998).

${ }^{12}$ C. A. Balanis, Advanced Electromagnetics (John Wiley \& Sons, New York, 1989).

${ }^{13}$ D. K. Cheng, Fundamentals of Engineering Electromagnetics, AddisonWesley Publishing.

${ }^{14} \mathrm{G}$. N. Watson, A Treatise on the Theory of Bessel Functions (Cambridge University Press, London, 1922).

${ }^{15} \mathrm{H}$. Irak, "Modeling RF heating of active implantable medical devices during MRI using safety index," M.Sc. thesis, Bilkent University, 2007.

${ }^{16}$ P. Nordbeck, I. Weiss, P. Ehses, O. Ritter, M. Warmuth, F. Fidler, V. Herold, P. M. Jakob, M. E. Ladd, H. H. Quick, and W. R. Bauer, "Measuring RF-induced currents inside implants: Impact of device configuration on MRI safety of cardiac pacemaker leads," Magn. Reson. Med. 61, 570578 (2009).

${ }^{17}$ H. Graf, U. A. Lauer, A. Berger, and F. Schick, "RF artifacts caused by metallic implants or structures which get more prominent at $3 \mathrm{~T}$ : An in vitro study," Magn. Reson. Imaging 23, 493-499 (2005). 
${ }^{18}$ J. P. Hornak, J. Szumowski, and R. G. Bryant, "Magnetic field mapping," Magn. Reson. Med. 6, 158-163 (1988).

${ }^{19}$ B. Akin, Y. Eryaman, and E. Atalar, "A method for phantom conductivity and permittivity measurements," 26th Annual ESMRMB Meeting, Antalya, Turkey (2009)

${ }^{20}$ G. H. Glover, C. E. Hayes, N. J. Pelc, W. A. Edelstein, O. M. Mueller, H. R. Hart, C. J. Hardy, M. O'Donnell, and W. D.

Barber, "Comparison of linear and circular polarization for magnetic resonance imaging,” J. Magn. Reson. 64, 255-270 (1985).

${ }^{21}$ P. A. Bottomley, A. Kumar, W. A. Edelstein, J. M. Allen, and P. V. Karmarkar, "Designing passive MRI-safe implantable conducting leads with electrodes," Med. Phys. 37, 3828 (2010).

${ }^{22}$ W. Kainz, G. Neubauer, R. berbacher, F. Alesch, and D. D. Chan, "Temperature measurement on neurological pulse generators during MR scans," Biomed. Eng. Online 1, 2 (2002). 